\title{
Bibliotecas universitarias de salud en el Perú
}

\author{
Julio César Olaya Guerrero \\ Departamento Académico de Bibliotecología y \\ Ciencias de la Información
}

\section{Introducción}

En los últimos años, las Bibliotecas Universitarias en el pais han pasado de los catálogos manuales a los sistemas automatizados de búsqueda bibliográfica mediante una interfaz Web. Esto permite la consulta en línea de las bases de datos.

En las universidades peruanas, los docentes, estudiantes, investigadores utilizan la tecnologia para acceder a nuevos conocimientos.

La satisfacción de las necesidades de información existentes en una comunidad de usuarios, ha llevado a la aplicación de nuevas y mejores tecnologías, así como el uso de nuevos enfoques administrativos con el fin de brindar un servicio de calidad. Asimismo la Hamada "Explosión de la Información", ha ocasionado que el procesamiento de esta involucre el uso de mejores recursos (humanos y técnicos) para un apropiädo hivelde afalisisis que cubra fas" expectativas y permita un acceso eficaz y efectivo a los fondos documentales de una institución o fuera de ella.

\section{Bibliotecas Universitarias de Salud en el Peri}

En nuestro país, las bibliotecas universitarias, organismos descentralizados, centros de investigación en salud proveen de información especializada a los médicos, estudiantes de medicina, investigadores y personas interesadas en el campo de la salud, para desarrollar sus trabajos de campo, tesis.

BIREME (Brasil) ha generado la Guía para el desarrollo de la biblioteca virtual en salud en el año 1999. Los principales lineamientos son: 
Acceso a literatura científico técnica.

Acceso a Directorios.

Apoyo a los procesos.

Apoyo a la educación en salud.

$\diamond$ Diseminación selectiva de información, Descriptores, Seminarios.

La biblioteca virtual nos permite acceder a información desde puntos remotos, sin necesidad de tenerla físicamente. Incorpora avances tecnológicos a partir de los principios de la realidad virtual; es una modalidad que se inserta en los servicios de información especializada, ya que administra el acceso a información remota que se encuentra en colecciones que poseen otros puntos de servicio, pero a la cual se puede acceder mediante las telecomunicaciones. Hasta el momento esta modalidad se da sobre temas muy especificos.

Según Abel Laerte, la idea de la Biblioteca virtual en salud es contribuir a la unión de la sociedad hacia la sociedad de la información, lo cual se rige por un principio que es la búsqueda de la equidad en acceso de la información, que es condición para dirigir una Unidad de salud. '

Podemos destacar a la Biblioteca de Biomédicas de la Universidad Nacional San Agustín de Arequipa, registra en forma detallada los recursos y servicios de información que ofrece a la comunidad de usuarios. Su web es: http://www.unsa.edur.pelbiblio/biomédicas

La Universidad Peruana Cayetano Heredia, a través de la DUIICT ofrece una variedad de recursos en línea en salud:

Bases de datos Enlaces a:

\begin{tabular}{ll}
\hline$\diamond$ LILACS & $\diamond$ Bibliotecas \\
$\diamond$ PUBMED & $\diamond$ REPEBIS \\
$\diamond$ PROQUEST & $\diamond$ Revistas en línea
\end{tabular}

Asimismo enlaces a Ministerios de salud, directorios de médicos especialistas. Pueden acceder mediante: http://www.upch.edu.pe/DUIICT

Laerte Packer, Abel. "Modelo de Biblioteca Virtual en salud para América Latina”. Common News, $n^{\circ} 36,2001$, p. 12. 
Otro enlace de gran importancia para los investigadores constituye Ia Universidad Nacional Mayor de San Marcos, permite acceder a información especializada en ciencias de la salud mediante bases de datos PROQUEST. También cuenta con su biblioteca virtual en salud, que reúne revistas médicas $y$ de areas a fines. Su web es: http:/www.unmsm.edu.pe

La biblioteca de la Facultad de Medicina de la Universidad San Martín de Porres nos ofrece una serie de recursos y servicios de información: catálogo bibliográfico, hemeroteca virtual, multimedia, links especializados, biblioteca virtual. El icono de biblioteca virtual de la Facullad presenta enlaces a páginas médicas, bases de datos EBSCO, PROQUEST, acceso a textos completos, diccionarios, enciclopedias. Se accede mediante: http:// www.medicina.usmp.edu.pe y luego click en el icono biblioteca.

La Universidad Particular Antenor Orrego (UPAO) suscribió recientemente un contrato con la companfa EBSCO para implementar bibliotecas virtuales. Este contrato permitira acceder a miles de artículos de investigación. La comunidad estudiantil de la UPAO cuenta con 29 computadoras de la biblioteca virtual de la Facultad de Medicina Humana. ${ }^{2}$

Si desea consultar las diferentes bibliotecas universitarias del Perú y del mundo puede acceder al website: http://www.universia.edu.pe portal de los universitarios y luego click en el ícono bibliotecas.

\section{Conclusiones}

Las Bibliotecas Universitarias contribuyen a la investigación académica de los docentes, estudiantes de medicina e investigadores. En el caso de la biblioteca virtual en salud es un potencial que permite tener información actualizada en forma permanente.

La biblioteca virtual en salud es un reto que debe difundirse en todas las Facultades de Medicina de nuestro pais. El desafio recién empieza!!!.

2 Bibliolecas virtuales en UPAO. Información disponible en http:/www.universia,edu.pe Consultado: 30 de marzo de 2002. 


\section{BIBLIOGRAFÍA}

ABADAL FALGUERAS, Ernest. "Las Bases de datos en España a principios del siglo XXI". En El Profesional de la información, 2001, diciembre, v.10, n.12, pp. 8-12.

BARBER, Elsa. "Factores incidentes en los resultados de la automatización bibliotecaria: una indagación sobre experiencias de países en desarrollo". En información, Cultura y Sociedad, 2000, n. 3, pp. 47-60.

BIBLIOTECA REGIONAL DE MEDICINA (BIREME). Consultado el 30 de marzo del 2002. http://www.bireme.br

BUSTELO, Carlota. "Tendencias en la gestión del conocimiento, la documentación y el conocimiento en las organizaciones". En El Profesional de la Información, 2001, diciembre, v.10, n. 12, pp. 4-7.

FERRAN, Núria. "Bases de datos bibliográfica y XML: el caso Medline”. En El Profesional de la Información, 2001, octubre, v.10, n.10, pp. 22-24.

GUERRERO BOTE, Vicente Pablo. "Inteligencia Artificial y Documentación". En Investigación Bibliotecológica, v. 15, n. 30, 2001, pp. 65-95.

HANSEN, Carol. "The internet navigator; An online internet course for dis tance Learners". En Library Trends, 2001, v.50, n. 1, pp. 58-72.

LAERTE, Abel. "Modelo đo-Bibliotecal Virtual ensalud para América Latina". En: Common News n 36; p. 12 trerso"

MOUNTAIN, David; Raper, Jonathan. "Positioning techniques for locationbased services: characteristics and limitations of proposed solutions": En Aaslib Proceedings, 2001, novembrer/december, v.53, n. 10, pp. $404-412$.

SALVADOR OLIVÁN, Juan Antonio; Francisco VIDAL BORDÉS. "Evaluación del rendimiento de los motores de búsqueda en la recuperación de la información en la WWW". En Documentación de las Ciencias de la Información, 2000, n.23, pp. 93-108.

SEDIC. Consultado el 30 de marzo del 2002. http://www.sedic.es

UNIVERSIDAD NACIONAL MAYOR DE SAN MARCOS. Consultado el 31 de marzo del 2002. http://www.unmsm.edu.pe

UNIVERSIA-PERU. Consultado el 27 de marzo del 2002. http:// www.universia.edu.pe 